NASZA DERMATOLOGIA Online OUR DERMATOLOGY Online

Source of Support: Nil

Competing Interests: None declared

\section{ANTINUCLEAR ANTIBODY LEVELS IN POLYMORPHIC LIGHT ERUPTION AND THEIR RELATION TO THE SEVERITY OF DISEASE}

\author{
Iffat Hassan ${ }^{1}$, Peerzada Sajad ${ }^{1}$, Sabiya Majid ${ }^{2}$, Hamid Bashir ${ }^{2}$ \\ ${ }^{1}$ Department of Dermatology, STD and Leprosy, Government Medical College, \\ Srinagar (University of Kashmir), $J \& K$, India \\ ${ }^{2}$ Department of Biochemistry, Government Medical College, Srinagar (University of \\ Kashmir), $J \& K$, India
}

Corresponding author: Associate Professor Iffat Hassan

hassaniffat@gmail.com

\begin{abstract}
Introduction: PLE is an idiopathic photodermatosis characterised by a polymorphic eruption ranging from papulo-vesicular lesions to large plaques, located predominantly in a photoexposed distribution. It is an acquired disease and is the most common idiopathic photodermatosis. It is characterised by recurrent abnormal delayed reaction to sunlight. PLE is the most common idiopathic photodermatosis, the prevalence of which has been estimated to be around 10-20\% in USA, England and Ireland. Previous studies have shown elevated levels of ANA in 2.9-19\% of patients with PLE.

Aim: The aim of our study was to determine the frequency of ANA positivity in a cohort of patients of ethnic kashmiri origin with Polymorphic light eruption and to examine whether there is any relation of their levels with the severity of disease.

Methods: Patients with Polymorphic light eruption clinically who attended the Outpatient Deptt. of Dermatology GMC Srinagar were referred to the Deptt. of Biochemistry GMC Srinagar where patients blood samples were analysed for ANA index by ELISA method (BRIO SEAC ITALY).

Results: Our study consisted of 36 patients (with 23 males and 13 females with age group ranging between 15-65 years) presenting with typical clinical features of PLE without associated autoimmune connected tissue diseases like discoid lupus erythematosus or systemic lupus erythematosus and 20 healthy age and sex matched controls. Two patients ( 1 male and 1 female) showed positive results and 1 patient (female) showed equivocal results. Among the control group one patient showed ANA positivity. Thus total frequency of ANA positivity of of 5.55\% was observed among the cases and 5\% among the controls with frequency of $4.34 \%$ in males and $7.69 \%$ in females.

Conclusions: ANA levels were found in 5.55\% of patients with PLE, however there is no relation between the levels of ANA in PLE and with the severity of disease ( $\mathrm{p}$ value $>0.05$ ).
\end{abstract}

Key words: antinuclear antibodies; polymorphic light eruption; discoid lupus erythematosus; ANA index

Abbreviations and acronyms: ANA (antinuclear antibodies), PLE (polymorphic light eruption), DLE (discoid lupus erythematosus), AI (ANA index).

\section{Introduction}

PLE is an idiopathic photodermatosis characterised by a polymorphic eruption ranging from papulo-vesicular lesions to large plaques, located predominantly in a photoexposed distribution. It is an acquired disease and is the most common idiopathic photodermatosis. It is characterised by recurrent abnormal delayed reaction to sunlight ranging from erythematous papules, papulovesicles and plaques to erythema multiforme like lesions on light exposed areas. Within any one patient only one clinical form is consistently manifested [1-3].

The condition is seen most commonly in parts of the world with four seasons and it is most commonly triggered by springtime sun exposure. The rash occurs on the sun exposed parts of the body, usually 1-4 days after exposure. 
The sensitivity of the skin and sensitivity of the rash gradually lessens as the spring changes into summer and skin becomes adjusted to light. PLE affects men and women, adults and children equally however the problem typically begins between the age of 20-35 years. (Native Americans have a high rate of PLE and there is some evidence of genetic predisposition) [4,5]. Previous studies have shown elevated levels of ANA in $2.9-19 \%$ of patients with PLE [6,7]. The prevalence of autoimmune diseases in PLE has been found to be as high as $15 \%$ in some studies and even higher (22\%) if hypothyroidism and non-toxic goitre were considered as the autoimmune processes. This is more than the estimated prevalence of $5-7 \%$ of the autoimmune diseases in the general population and indicates that the patient's with PLE have an increased risk of contracting an immunological disorder, although cases of lupus erythematosus didn't outnumber those of any other autoimmune disease [8]. Epstein has postulated that PLE is a cell mediated, delayed type hypersensitivity response to a sunlight-induced neoantigen. Another pathogenic pathway postulated is immunosuppression by ultravoilet radiation (due to genetic predisposition) resulting in increased recognition of antigens. This could be either due to resistance of Langerhan's cells to depletion by very intense ultravoilet radiation exposure and a reduced capacity to handle free radical insult through genetically determined glutathione depletion $[9,10]$.

\section{Material and Methods (Fig. 1, 2)}

Our study consisted of 36 patients and 20 healthy age and sex matched controls who attended the outpatient Department of Dermatology, STD and Leprosy an associated hospital of GMC Srinagar. Out of 36 patients; 23 were males and 13 were females. Their age group ranged between $15-$ 65 years (2). The method used to measure ANA index was ELISA BRIO SEAC (ITALY). The patients having classical clinical features of PLE and without other criteria suitable to the diagnosis of lupus erythematosus were regarded as having PLE and were included in the study. All the patients were ethnic kashmiris. An assessment of the patients present history, and personal history included questions about photosensitivity, allergy, autoimmune diseases, drug intake and a detailed analysis of the individual PLE lesions (Morphology, symptoms, latency period between sun exposure and beginning of skin rash, duration of the disease and resolution with or without scarring. Particular attention was paid to any symptoms of collagen vascular disorders in particular lupus erythematosus. In Addition, the patients were examined for any physical signs of Lupus erythematosus as defined by the 1982-revised ARA criteria.

\section{Exclusion criteria:}

1. The patients having clinical manifestation of lupus erythematosus or other autoimmune diseases were excluded from the study.

2. Non-kashmiris:

Controls: Control population consisted of 20 individuals (12 males, 8 females) without clinical features of PLE.

The limitations of the study were low patient number and the basis of the diagnosis of PLE was clinical. Informed consent was obtained from each patient.
The statistical method used for drawing the inferences was one -sample t-test.

The study was approved by the ethical committee of the hospital.

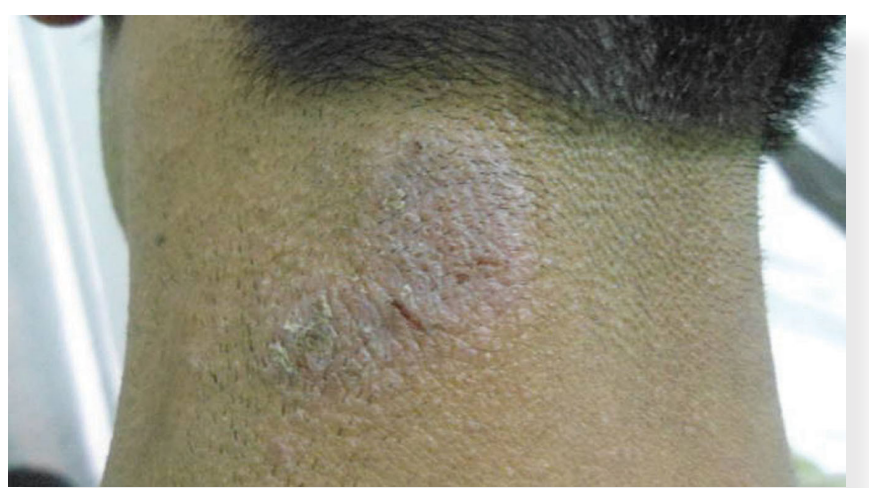

Figure 1. Plaque type PLE lesions on the nape of neck

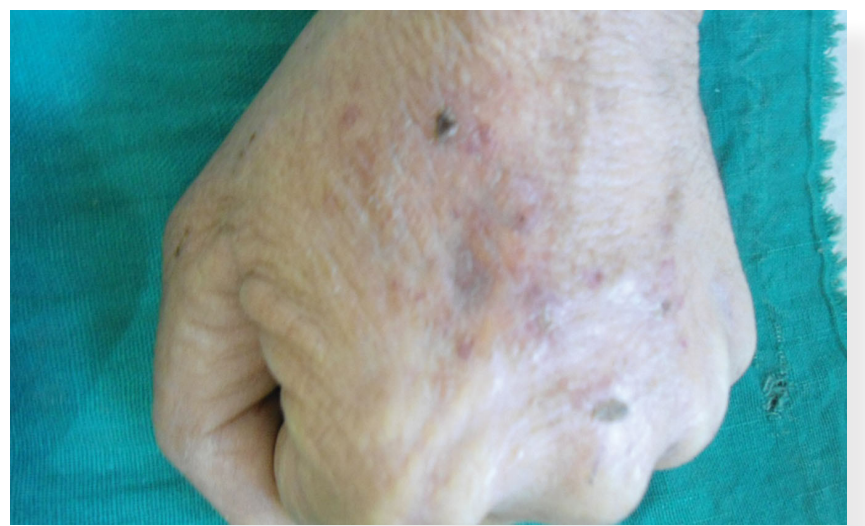

Figure 2. Erythema multiforme-like lesions and voilaceous papules over the dorsum of hands

\section{Results}

Two patients ( 1 male and 1 female) showed positive results and 1 patient (female) showed equivocal results. Among the control group one patient showed ANA positivity. Thus total frequency of ANA positivity of of $5.55 \%$ was observed. Among the cases and 5\% among the controls with frequency of $4.34 \%$ in males and $7.69 \%$ in females (Tabl. I).

\section{Disussion}

PLE is an idiopathic photodermatosis characterised by a polymorphic eruption ranging from papulo-vesicular lesions to large plaques, located predominantly in a photoexposed distribution. It is an acquired disease and is the most common idiopathic photodermatosis. It is characterised by recurrent abnormal delayed reaction to sunlight ranging from erythematous papules, papulovesicles and plaques to erythema multiforme like lesions on light exposed areas. Within any one patient only one clinical form is consistently manifested [1-3]. Our study consisted of 36 patients who were clinically diagnosed as cases of polymorphic light eruption who were compared with 20 healthy age and sex matched controls. The most common type of clinical presentation was the micropapular type of lesions followed by the plaque, erythema multiforme and eczematous type of lesions. 


\begin{tabular}{|l|l|l|l|l|l|}
\hline & Total & M & F & +ives & -ives \\
\hline CASES & 36 & 23 & 13 & 3 & 33 \\
\hline CONTROLS & 20 & 12 & 8 & 1 & 19 \\
\hline
\end{tabular}

Table I. Incidence of toe and finger nail infections $(n=75)$

Total cases studied $=36$

Normal range: ELISA METHOD

<1.0AI- NEGATIVE; 1.0-1.1AI=EQUIVOCAL; >1.1AI = (+IVE)

AI=ANA INDEX (Ratio of sample observance with cutoff)

The severity was clinically assessed on the basis of extent of involvement of photoexposed areas, time taken to resolution, intensity of pruritus and frequency of recurrences. All the patients were ethnic kashmiris. An assessment of the patients present history, and personal history included questions about photosensitivity, allergy, autoimmune diseases, drug intake and a detailed analysis of the individual PLE lesions (morphology, symptoms, latency period between sun exposure and beginning of skin rash, duration of the disease and resolution with or without scarring. Particular attention was paid to any symptoms of collagen vascular disorders in particular lupus erythematosus. In Addition, the patients were examined for any physical signs of Lupus erythematosus as defined by the 1982-revised ARA criteria. There was no significant difference in the ANA positivity rate between cases and controls and also of the levels of ANA with the severity of disease.

\section{Conclusion}

Positive ANA levels were found in $5.55 \%$ of patients with PLE, which is consistent with the results of earlier studies, however there is no relation between the levels of ANA in PLE and with the severity of disease.

\section{REFERENCES}

1. Stratigos AJ, Antoniou C, Katsambas AD: Polymorphic light eruption. J Eur Acad Dermatol Venereol. 2002;16:193-206.

2. Naleway AL: Polymorphic light eruption. Int J Dermatol. 2002;41:377-83.

3. Epstein JH: Polymorphic light eruption. Phototesting technique studies. Arch Dermatol. 1962;85:502-4.

4. Norris PG, Morris J, McGibbon DM, Chu AC, Hawk JL: Polymorphic light eruption: an immunopathological study of evolving lesions. Br J Dermatol. 1989;120:173-83.

5. Morrison WL, Stern RS: Polymorphic light eruptions: A common reaction uncommonly recognised. Acta Derm Venereal. 1982;62:237-40.

6. Norris PG, Hawk JLM: The acute idiopathic photodermatosis. Sem Dermatol. 1990;9:32-8.

7. Sinha AA, Lopez MT, Mcdevitt HD: Autoimmune diseases: The failure of self tolerance. Science. 1990:248;1380-7.

8. Young AR: Cumulative effects of ultraviolet radiation on the skin.Cancer and Photoageing. Semin Dermatol. 1990;9:25-31.

9. Fisher MS, Kripkle ML: Suppressor T- lymphocytes control the development of primary skin cancers in ultra-voilet irradiated mice. Science. 1982;216:1133-4.

10. Frain-Bell W: Cutaneous photobiology. Oxford: Oxford University Press; 1985. p. 24-59.

Copyright by Iffat Hassan et al. This is an open access article distributed under the terms of the Creative Commons Attribution License, which permits unrestricted use, distribution, and reproduction in any medium, provided the original author and source are credited. 